\title{
STAT1 gene mutation is not implicated in upper aerodigestive cancers
}

\author{
Polat Dura ${ }^{1}$, Rene H. M. te Morsche ${ }^{1}$, Martin Lacko ${ }^{2}$, Mihai G. Netea ${ }^{1}$, Jos W. M. van der Meer ${ }^{1}$, \\ Joost P. H. Drenth ${ }^{1}$, Wilbert H. M. Peters ${ }^{{ }^{*}}$ \\ ${ }^{1}$ Departments of Gastroenterology and Internal Medicine, Radboud University Nijmegen Medical Center, Nijmegen, The Netherlands \\ ${ }^{2}$ Department of Otorhinolaryngology, Maastricht University Medical Center, Maastricht, The Netherlands \\ Email: ${ }^{*}$ w.peters@mdl.umcn.nl
}

Received 2 December 2011; revised 13 February 2012; accepted 17 February 2012

\begin{abstract}
Autosomal Dominant Chronic Mucocutaneous Candidiasis (AD-CMC) is characterized by defective $T$ cell immunity, leading to fungal infections limited to mucosal surfaces. Recently it was discovered that mutations in the coiled-coil (CC) domain of STAT1 are the cause of AD-CMC. STAT1 deficiency has been implicated in experimental models of oesophageal cancer (EC) and head and neck carcinoma (HNC). Both carcinoma types are prevalent among CMC patients. Consequently, we postulated that the same mutation in the STAT1 gene triggering ADCMC, could also be involved in oesophageal or head and neck carcinogenesis. However we failed to identify the c.820C $>$ T mutation in the STAT1 CC domain in 3 cohorts of Dutch Caucasian origin: being 351 EC patients, 325 HNC patients and 309 controls. Although it seems valuable to investigate the relationship between AD-CMC and upper aerodigestive neoplasms, the c.820C $>\mathrm{T}$ mutation in the STAT1 gene does not seem implicated in EC and HNC aetiology.
\end{abstract}

Keywords: STAT1; Signal Transducers and Activators of Transcription; Chronic Mucocutaneous Candidiasis; Genetic Mutation; Esophageal Carcinoma; Head and Neck Carcinoma

\section{INTRODUCTION}

Signal transducers and activators of transcription (STAT) are dormant cytoplasmic proteins that upon activation regulate a wide variety of cellular processes, such as immune regulation, apoptosis, differentiation and proliferation [1,2]. In humans, seven STATs (1-4, 5a, 5b, 6) have been discovered [2]. Phosphorylation of tyrosine residues precedes activation of STAT and is achieved through two main signalling routes: the growth factor-

*Corresponding author. and cytokine-signalling pathway [1,2]. Growth factor signalling occurs primarily via the Epidermal Growth Factor Receptor (EGFR). Conversely, cytokine activation of Janus Tyrosine Kinases (JAKs), is an effective alternative $S T A T$ activation pathway.

Dysregulation of these pathways is frequently observed in primary malignant tumours. Particularly STAT1 and STAT3 play an important role in carcinogenesis, and both are well studied in a range of malignancies [3]. STAT1 target genes include tumour suppressor genes, whereas target genes of STAT3 are oncogenes that stimulate cell cycle progression and inhibit apoptosis [3]. However, the exact role of STAT in oncogenesis remains complex and is incompletely understood. This is illustrated by the fact that STAT1 and STAT3 are sometimes expressed simultaneously [3].

STAT1 appears also to be involved in inflammatory and immunological diseases as it regulates immune effector genes [4], and plays for example a causative role in chronic mucocutaneous candidiasis (CMC) [5]. CMC can be divided into several diseases: CMC with Autoimmune Polyendocrinopathy-Candidiasis-Ectodermal Dystrophy (APECED), CMC without APECED but associated with primary hypothyroidism, and sporadic CMC [6]. APECED has an autosomal recessive manner of inheritance due to mutations in the AIRE gene, which expresses a protein that acts as a transcriptional activator [7]. The disease is characterized by the presence of autoreactive $\mathrm{T}$ lymphocyte responses, and subsequently neutralizing autoantibodies against cytokines of the Th17 family such as IL-17 and IL-22 [7], leading to fungal infections (mainly Candida) limited to mucosal surfaces. The second syndrome is inherited through an autosomal dominant pattern. Recently it was discovered that mutations in the $C C$ domain of STAT1 are the cause of autosomal dominant CMC (AD-CMC) [5], resulting in defective type 1 and type 17 helper $\mathrm{T}$ cell immunity. The c.820C > T mutation in the STAT1 gene results in an arginine to tryptophan amino acid substitution at codon 274 [5] and leads to a defective CC domain of the STAT1 
protein. It has been shown that this defect results in defective IL-12R and IL-23R signalling pathways, with absent production of IL-17, IL-22 and IFN $\gamma$ [5].

STAT1 deficiency has been implicated in experimental models of oesophageal cancer (EC) and head and neck cancer (HNC) $[8,9]$. Both carcinoma types are prevalent among CMC patients [6,10]. Interestingly, Veerdonk et al. reported that 3 out of their $14 \mathrm{AD}-\mathrm{CMC}$ patients also suffered from EC or HNC [5].

Consequently we postulated that the same mutation in the STAT1 gene, triggering AD-CMC as described by Veerdonk et al., could also be involved in oesophageal or head and neck carcinogenesis (Figure 1).

\section{MATERIALS AND METHODS}

Three cohorts of Dutch Caucasian origin were studied: 351 patients with oesophageal cancer [11], 325 patients with head and neck carcinoma [12], and a group of 309 healthy controls, recruited from the same geographical area as the patients [11]. A 60 bp region of exon 10 of the STAT1 gene, including the c.820C > T mutation, was examined by means of PCR followed by High Resolution Melting analysis. After using the forward primer 3'CTTGTGTCTTCCCAGGTTCA-5', the reverse primer 3'-CCAATTCCTCCAACTTTTTAAGC-5' and EvaGreen (Biotium, Hayward, CA) as the fluorescent dye in the PCR, melting curves from $65^{\circ} \mathrm{C}$ to $95^{\circ} \mathrm{C}$ with a ramp rate of $0.1^{\circ} \mathrm{C} / 10$ seconds were obtained with the $\mathrm{CFX} 96^{\mathrm{TM}}$ Real-Time PCR Detection System (Biorad Laboratories, Hercules, CA). Melting curves were analyzed using the
Precision Melt software (Biorad). Samples with deviant curves were sequenced.

\section{RESULTS AND DISCUSSION}

We failed to identify the c.820C $>\mathrm{T}$ mutation in the STAT1 CC domain in our two cohorts of 676 patients with upper aerodigestive cancer. Likewise, the mutation was also absent in 309 healthy controls. Demographics of patients and controls are shown in Table 1.

It has been suggested that oral or oesophageal cancer in APECED-CMC may be induced by the production of carcinogens by $C$. albicans [13]. Although EC and HNC are reported in $\mathrm{AD}-\mathrm{CMC}[5,6]$, a clear correlation has not been proven, and the possible mechanism remains unclear. However, as with APECED-CMC, prolonged exposure to $C$. albicans infections in the upper GI tract

Table 1. Characteristics of patients with esophageal cancer, head and neck cancer and controls.

\begin{tabular}{lccc}
\hline \multicolumn{1}{c}{ Characteristics } & Patients & \multicolumn{1}{c}{ Controls } \\
\hline & EC & HNC & \\
Number (\% of total) & $351(100 \%)$ & $325(100 \%)$ & $309(100 \%)$ \\
Age (yrs; mean \pm SD) & $65.0 \pm 10.9$ & $61.8 \pm 11.6$ & $42.0 \pm 16.3$ \\
Gender & & & \\
Male & $283(80.6 \%)$ & $260(80.0 \%)$ & $188(60.9 \%)$ \\
Female & $68(19.4 \%)$ & $65(20.0 \%)$ & $121(39.1 \%)$ \\
\hline
\end{tabular}

EC, esophageal carcinoma; HNC, head and neck carcinoma.

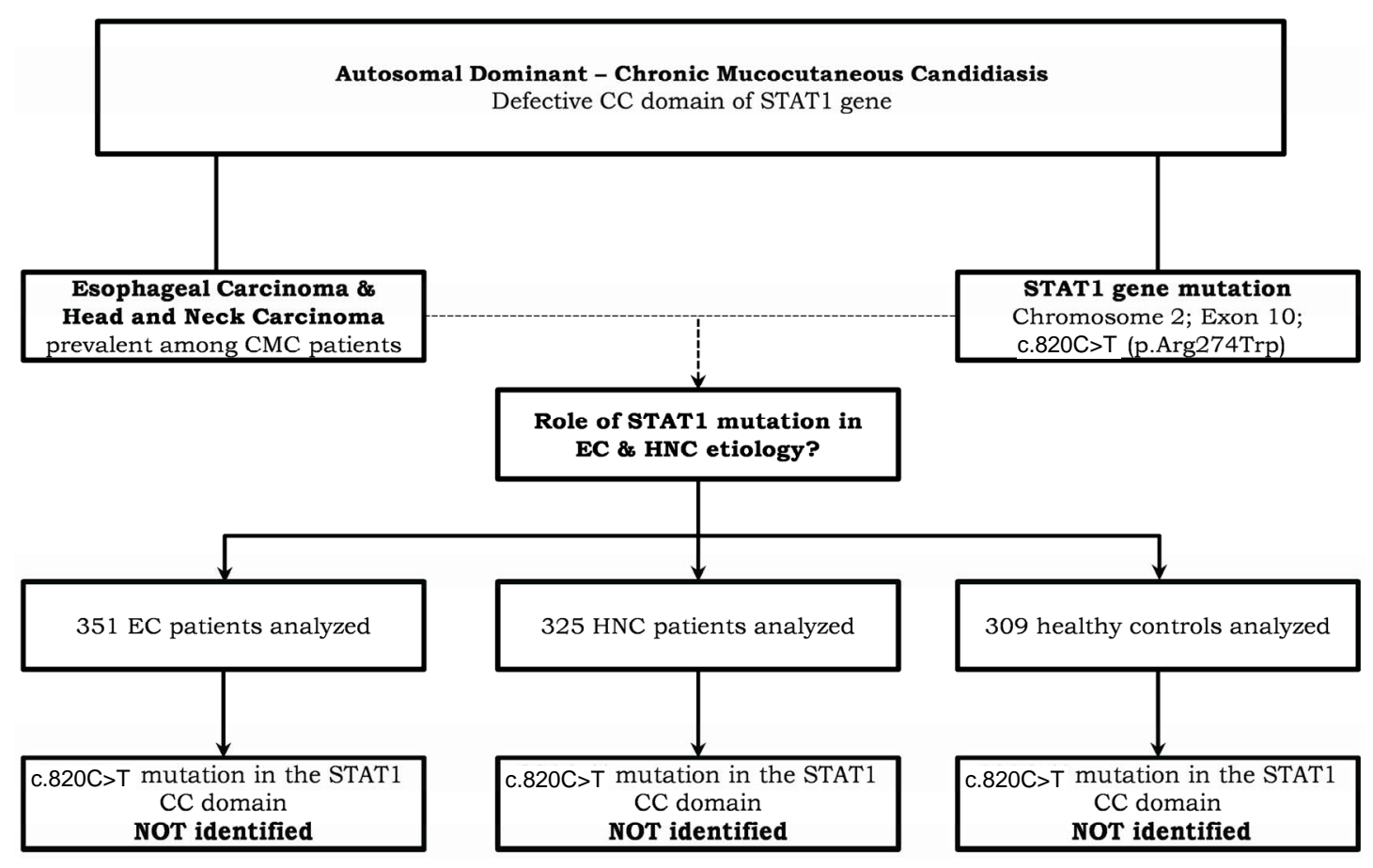

Figure 1. Flowchart. 
can be an etiological factor [14]. IgA deficiency might be another immunological clarification for EC and HNC in AD-CMC. IgA deficiency, linked with neoplasm development [14], has also been reported in APECED negative CMC patients $[14,15]$. Although there is no known correlation with EC and HNC, IgA deficiency is reported to influence oesophageal dysplasia [16].

Our findings do not support a role of the c.820C $>\mathrm{T}$ mutation in the STAT1 gene with oesophageal or head and neck carcinogenesis. The only mutation that was found twice in both patient groups and twice in the control group, was the c.796G $>$ A mutation (rs41473544). However, its occurrence in both patients and controls suggests no pathophysiological relevance.

Furthermore, there is no other clear evidence that the STAT1 mutation is involved in EC risk, although there are reports that STAT1 activation induces apoptosis in EC cells in vitro [17,18], and loss of STAT1 activity was suggested to result in EC progression [8]. To our knowledge no case-control or GWAS studies examining the role of STAT1 mutations in EC and HNC are known. For HNC as well, most of the STAT1 related basic research concentrates on biomarker and therapeutic potential [19, 20].

In conclusion, it seems valuable to investigate the possible relationship between oesophageal and head and neck malignancy and APECED-CMC and AD-CMC. However, pursuing the c.820C $>$ T mutation in the STAT1 gene as an etiological factor for the development of EC/HNC in the absence of CMC does not seem worthwhile. Moreover, the STAT1 overexpression may not be independent, as STAT3 activation seems to occur simultaneously in a wide range of neoplasms. Consequently, adding the fact that STAT3 is an oncogene, haplotyping STAT1 and STAT3 genes in both carcinoma groups may be a more promising approach.

\section{ACKNOWLEDGEMENTS}

M. G. Netea was supported by a Vici Grant of the Netherlands Organization for Scientific Research.

All authors declare no conflicts of interest and have participated appropriately to take public responsibility.

\section{REFERENCES}

[1] Adamkova, L., Souckova, K. and Kovarik, J. (2007) Transcription protein STAT1: Biology and relation to cancer. Folia Biologica, 53, 1-6.

[2] Yang, J. and Stark, G.R. (2008) Roles of unphosphorylated STATs in signaling. Cell Research, 18, 443-451. doi:10.1038/cr.2008.41

[3] Bowman, T., Garcia, R., Turkson, J. and Jove, R. (2000) STATs in oncogenesis. Oncogene, 19, 2474-2488. doi:10.1038/sj.onc.1203527
[4] Nishibori, T., Tanabe, Y., Su, L. and David, M. (2004) Impaired development of $\mathrm{CD} 4^{+} \mathrm{CD} 25^{+}$regulatory $\mathrm{T}$ cells in the absence of STAT1: Increased susceptibility to autoimmune disease. Journal of Experimental Medicine, 199, 25-34. doi:10.1084/jem.20020509

[5] van de Veerdonk, F.L., Plantinga, T.S., Hoischen, A., Smeekens, S.P., Joosten, L.A., Gilissen, C., et al. (2011) STAT1 Mutations in Autosomal Dominant Chronic Mucocutaneous Candidiasis. New England Journal of Medicine, 365, 54-61. doi:10.1056/NEJMoa1100102

[6] Koch, D., Lilic, D. and Carmichael, A.J. (2009) Autosomal dominant chronic mucocutaneous candidiasis and primary hypothyroidism complicated by oesophageal carcinoma. Clinical and Experimental Dermatology, 34, 818-820. doi:10.1111/j.1365-2230.2009.03561.x

[7] Bjorses, P., Halonen, M., Palvimo, J.J., Kolmer, M., Aaltonen, J., Ellonen, P., et al. (2000) Mutations in the AIRE gene: Effects on subcellular location and transactivation function of the autoimmune polyendocrinopathy-candidiasis-ectodermal dystrophy protein. American Journal of Human Genetics, 66, 378-392. doi:10.1086/302765

[8] Watanabe, G., Kaganoi, J., Imamura, M., Shimada, Y., Itami, A., Uchida, S., et al. (2001) Progression of esophageal carcinoma by loss of EGF-STAT1 pathway. Cancer Journal, 7, 132-139.

[9] Shim, S.H., Sung, M.W., Park, S.W. and Heo, D.S. (2009) Absence of STAT1 disturbs the anticancer effect induced by STAT3 inhibition in head and neck carcinoma cell lines. International Journal of Molecular Medicine, 23, 805-810.

[10] Firth, N.A., O’Grady, J.F. and Reade, P.C. (1997) Oral squamous cell carcinoma in a young person with candidosis endocrinopathy syndrome: A case report. International Journal of Oral and Maxillofacial Surgery, 26, 42-44. doi:10.1016/S0901-5027(97)80845-4

[11] Kristinsson, J.O., Van Westerveld, P., Te Morsche, R.H.M., Roelofs, H.M.J., Wobbes, T., Witteman, B.J., et al. (2009) Cyclooxygenase-2 polymorphisms and the risk of esophageal adeno- or squamous cell carcinoma. World Journal of Gastroenterology, 15, 3493-3497. doi:10.3748/wjg.15.3493

[12] Lacko, M., Roelofs, H.M.J., Te Morsche, R.H.M., Voogd, A.C., Oude Ophuis, M.B., Peters, W.H.M., et al. (2010) Genetic polymorphism in the conjugating enzyme UGT1A1 and the risk of head and neck cancer. International Journal of Cancer, 127, 2815-2821. doi:10.1002/ijc.25296

[13] Bockle, B.C., Wilhelm, M., Muller, H., Gotsch, C. and Sepp, N.T. (2010) Oral mucous squamous cell carcinoma-An anticipated consequence of autoimmune polyendocrinopathy-candidiasis-ectodermal dystrophy (APECED). Journal of the American Academy of Dermatology, 62, 864-868. doi:10.1016/j.jaad.2009.06.061

[14] Rosa, D.D., Pasqualotto, A.C. and Denning, D.W. (2008) Chronic mucocutaneous candidiasis and oesophageal cancer. Medical Mycology, 46, 85-91. doi:10.1080/13693780701616023

[15] Kalfa, V.C., Roberts, R.L. and Stiehm, E.R. (2003) The syndrome of chronic mucocutaneous candidiasis with selective antibody deficiency. Annals of Allergy Asthma 
and Immunology, 90, 259-264. doi:10.1016/S1081-1206(10)62152-7

[16] Joshi, N., Johnson, L.L., Wei, W.Q., Abnet, C.C., Dong, Z.W., Taylor, P.R., et al. (2006) Gene expression differences in normal esophageal mucosa associated with regression and progression of mild and moderate squamous dysplasia in a high-risk Chinese population. Cancer Research, 66, 6851-6860.

doi:10.1158/0008-5472.CAN-06-0662

[17] Kaganoi, J., Watanabe, G., Okabe, M., Nagatani, S. Kawabe, A., Shimada, Y., et al. (2007) STAT1 activation-induced apoptosis of esophageal squamous cell carcinoma cells in vivo. Annals of Surgical Oncology, 14, 1405-1415. doi:10.1245/s10434-006-9274-7

[18] Ichiba, M., Miyazaki, Y., Kitamura, S., Kiyohara, T.,
Shinomura, Y. and Matsuzawa, Y. (2002) Epidermal growth factor inhibits the growth of TE8 esophageal cancer cells through the activation of STAT1. Journal of Gastroenterology, 37, 497-503. doi:10.1007/s005350200077

[19] Laimer, K., Spizzo, G., Obrist, P., Gastl, G., Brunhuber, T., Schafer, G., et al. (2007) STAT1 activation in squamous cell cancer of the oral cavity: A potential predictive marker of response to adjuvant chemotherapy. Cancer, 110, 326-333. doi:10.1002/cncr.22813

[20] Nikitakis, N.G., Siavash, H. and Sauk, J.J. (2004) Targeting the STAT pathway in head and neck cancer: Recent advances and future prospects. Current Cancer Drug Targets, 4, 637-651. doi:10.2174/1568009043332736 